Journal of Neurology, Neurosurgery, and Psychiatry 1986;49:585-587

Short report

\title{
An assessment of the possible protective effect of allopurinol in acute stroke
}

\author{
ROBERT IANSEK, DAVID PACKHAM, BENJAMIN S ASPEY, \\ MICHAEL JG HARRISON
}

From the Department of Neurology, The Middlesex Hospital, London, UK

SUMMARY Allopurinol has been shown to have a protective effect on ischaemic tissue by the indirect prevention of excessive purine loss. This property was tested in the gerbil model of acute stroke. A total of 69 animals were pretreated with an intraperitoneal injection of either allopurinol $(50 \mathrm{mg} / \mathrm{kg})$ or sterile water and then subjected to unilateral ligation of the left common carotid artery under general anaesthesia. The clinical effect of the ligation was grouped into three categories of normal, mild to moderate defect (splayed leg, turning behaviour) and severe defect (death, nonresponsiveness and seizures). More normal animals and fewer severely affected animals were present in the allopurinol treated group compared to controls, but only at 2 to 4 hours after carotid ligation $(\mathrm{p}<0.05)$. Histological examination of brain tissue from the normal category failed to reveal any difference in subclinical ischaemic damage between the two groups. It was concluded that allopurinol may have a protective effect in acute stroke and that this property warrants further elucidation.

The causes of brain damage in cerebral ischaemia have been extensively investigated and a variety of mechanisms have been implicated. ${ }^{12}$ However, the role of purine nucleotides in cerebral infarction has been relatively neglected despite their central role in cell energy metabolism. Investigation of the role of purine nucleotides in ischaemic states involving other tissues has suggested that the extent of tissue damage may be partly related to depletion of such molecules during the ischaemic period, and that administered allopurinol may have a protective effect under such conditions. The explanation of these findings is as follows. Purine nucleotides constitute an important part of adenosine triphosphate (ATP), essential for the provision of intracellular energy. It has been established that tissue hypoxia results in a progressive depletion of tissue ATP proportional to the duration of the ischaemic injury. ${ }^{3}$ During this fall in intracellular ATP, excess quantities of purine degradation inter-

Address for reprint requests: Dr MJG Harrison, The Middlesex

Received 23 July 1985 and in revised form 9 October 1985. Accepted 10 October 1985 mediaries, such as inosine and hypoxanthine, have been demonstrated. ${ }^{4}$ Depletion of intracellular ATP and its degradation intermediaries, beyond a critical level, results in irreversible changes within the cell which result in an inability of the cell to regenerate ATP when the ischaemic conditions are reversed. ${ }^{35}$ Uric acid, the breakdown product of hypoxanthine, has also been shown to increase hypoxic conditions. Since the formation of uric acid is irreversible, its formation represents a relative loss of purine bases, which in turn limits the ability of the cell to resynthesise ATP. ${ }^{6}$ Purine nucleotides may be reconstituted during hypoxia, or following relief of hypoxia, by two mechanisms which differ in their energy requirements. De novo synthesis requires the utilisation of six high energy phosphate bonds. The alternative pathway of purine "salvage" involves the reutilisation of purine degradation products, particularly hypoxanthine, by the addition of ribose phosphate moiety requiring only one high energy phosphate bond. The salvage mechanism therefore represents a more efficient source of purines in conditions of energy depletion. Allopurinol, the xanthine oxidase inhibitor, has been shown to protect the cell 
against the effects of ischaemia by two mechanisms. ${ }^{67}$ Firstly by preventing the formation of uric acid it avoids irreversible loss of purine bases and by increasing circulating levels of hypoxanthine, via its effects on hepatic xanthine oxidase, it provides a source of purine reutilisation. Secondly, allopurinol inhibits de novo synthesis of purines. ${ }^{7}$ In this way it promotes purine salvage and thus may protect energy stores during periods of critical intracellular energy depletion. We wondered if depletion of purine nucleotides contributed significantly to cerebral damage particularly in oligaemic areas of brain tissue, in the same way as has been suggested for other tissues, such as the dog myocardium. ${ }^{8}$ In such a situation allopurinol might have some protective effect, reducing the final infarct area, or moderating the clinical deficit. We have tested this hypothesis in the gerbil stroke model.

\section{Method}

Sixty-nine mongolian gerbils of either sex weighing between 50 and 90 grams were used in this study. Thirty-four were pretreated with intraperitoneal injections of $50 \mathrm{mg} / \mathrm{kg}$ allopurinol $(10 \mathrm{mg} / \mathrm{ml}$ aqueous solution) and 35 with $5 \mathrm{ml} / \mathrm{kg}$ sterile water as controls. Injections were given at 12 hourly intervals on five separate occasions prior to surgery. Following the last injection, the animals were anaesthetised with diethyl ether (Analar, BDH) and, through a midline cutaneous incision, the left common carotid artery was exposed and permanently ligated with 5-0 silk. The skin was sutured and the animals were allowed to recover. The surgical procedure lasted 5-10 minutes. Their clinical state was monitored continuously for the first six to eight hours and then again at 24 hours after carotid occlusion. To facilitate an assessment of the clinical effects of allopurinol on cerebral ischaemia, the clinical status of both treatment groups was compared at 2-4 hours and at 24 hours. Disability at these times was graded as normal (no abnormalities apart from occasional ptosed eyelid), mild to moderate (splayed leg, turning behaviour) and severe (non-responsiveness, repeated fitting and death). Unfortunately clinical assessment was omitted in three animals (two allopurinol treated and one control) during the earlier period (2-4 hours).

At 24 hours after carotid occlusion, the animals were reanaesthetised with intraperitoneal pentobarbitone sodium (Sagatal, May and Baker) and killed by rapid intracardiac perfusion with $10 \%$ formol saline. The brains were removed and immersed in $30 \%$ sucrose in $10 \%$ formol saline for at least 7 days. Frozen sections $(50 \mu$ thick) were taken through the optic chiasm of brains from clinically normal animals with a freezing microtome, and stained with cresyl fast violet. The sections were then examined microscopically for histological evidence of ischaemic damage.

\section{Results}

The table shows a summary of the results. There were more normal animals in the allopurinol treated group
Table Summary of data

\begin{tabular}{llllll}
\hline \multirow{2}{*}{$\begin{array}{l}\text { Disability } \\
\text { grade }\end{array}$} & \multicolumn{2}{l}{ Allopurinol } & & \multicolumn{2}{l}{ Control } \\
\cline { 2 - 3 } \cline { 5 - 6 } \cline { 5 - 6 } & Early & Late & & Early & Late \\
\hline Normal & $25^{*}$ & 21 & & $15^{*}$ & 16 \\
Mild-moderate & 2 & 7 & & 2 & 5 \\
Severe & $6^{*}$ & 6 & & $16^{*}$ & 14 \\
Total & 33 & 34 & & 33 & 35 \\
\hline
\end{tabular}

*Significant difference $(\mathrm{p}<0.05)$.

than in the control group at both early ( $\overline{2}-\dot{4}$ hours) and late (24 hours) periods of observation; however, only at the early stage was this difference significant (Chi square $=5.14, p<0.05$ ). Similarly there were fewer animals severely affected in the allopurinol treated group than the control group for both early and late observation periods. Again only the difference at $2-4$ hours was significant (Chi square $=$ $5.52, \mathrm{p}<0.05$ ). There was no difference in the mild to moderate group between allopurinol and control animals at either period of observation. Five animals in the allopurinol treated group progressed to a worse grade over the period of observation to the second period at 24 hours and three animals progressed in the control group. This difference was not significant. In case there were any differences in the extent of subclinical ischaemic damage in the clinically normal groups these were subjected to histological examination. There was however evidence of ischaemic damage in all animals, consisting of patchy involvement of watershed regions of the cerebral cortex and cortical layers 3-5. No difference was apparent in the extent of ischaemic damage between the allopurinol and control groups on visual inspection.

\section{Discussion}

The results suggest that in the early period after carotid ligation allopurinol may have a protective effect on the extent of ischaemic damage which is not maintained at 24 hours. One reason for this difference could be that the effect of allopurinol had declined by 24 hours as no extra dose was administered after carotid ligation. This explanation is supported by experiments performed on dog myocardium, which showed that continued treatment with allopurinol was needed to prevent subsequent deterioration after induction of ischaemia, despite pretreatment with allopurinol. ${ }^{9}$ Unfortunately, it was not possible to compare the two groups directly at the two observation times of 2-4 hours and at 24 hours as the numbers differed owing to the failure to document the clinical status of three animals at 2-4 hours after carotid ligation. Furthermore, even though deterioration in clinical status over the 24 hours period occurred in more allopurinol 
treated animals, this was not significant. The conclusion that allopurinol has a protective effect must thus be made with caution, despite the statistical significance that was achieved. Further evaluation of these results are needed, as the degree of protection of allopurinol may be suggested by its continued administration after induction of ischaemia and by the concomitant infusion of hypoxanthine. This latter manoeuvre may be necessary because any rise in hypoxanthine in the serum will be equalled to a lesser degree by an increased urinary excretion, thus limiting any possible therapeutic effect of a high circulating level of this substrate. This latter point is important as maximum recovery from ischaemia would appear to be optimum following administration of hypoxanthine and allopurinol. ${ }^{6}$ To our knowledge the only assessment of the effect of allopurinol in acute stroke was directed toward the adenylate cyclase system. ${ }^{10}$ This study found that treatment of gerbils with allopurinol 30 minutes prior to ischaemia reversed the damage to adenylate cyclase elicited by prior 60 minute occlusion of both carotid arteries and 15 minute reflow. How these data relate to behavioural severity of ischaemic damage is unclear; however, the data do emphasise the protective effect of allopurinol in cerebral ischaemia and testify to the requirement of further assessment of a possible therapeutic role of allopurinol in acute stroke.
References

${ }^{1}$ Siesjo BK. Cerebral circulation and metabolism. $J$ Neurosurg 1984;60:883-908.

${ }^{2}$ Plum F. What causes infarction in ischaemic brain? The Robert Wartenberg Lecture. Neurology (Cleveland) 1983;33:222-33.

${ }^{3}$ Reibel DK, Rovetto MJ. Myocardial ATP synthesis and mechanical function following oxygen deficiency. Am J Physiol 1978;234:620-4.

${ }^{4}$ Imai S, Riley AL, Berne RM. Effect of ischaemia and adenine nucleotides in cardiac and skeletal muscle. Circ Res 1964;15:443-9.

${ }^{5}$ Jones CE, Crowell JW, Smith E. Significance of increased blood uric acid following extensive haemorrhage. Am J Physiol 1968;214:1374-7.

${ }^{6}$ Cunningham SK, Keaveny TV. Effect of a xanthine oxidase inhibition on adenine nucleotide degradation in haemorrhagic shock. Eur Surg Res 1978;10:305-13.

${ }^{7}$ Kelley WN, Wyngaarden JB. Effects of allopurinol on purine synthesis in cultured human cells. J Clin Invest 1970;49:602-9.

${ }^{8}$ De Wall RA, Vasko KA, Stanley EL, Kezdi P. Responses of the ischaemic myocardium to allopurinol. Am Heart $J$ 1971;82:362-79.

${ }^{9}$ Arnold WL, De Wall RA, Kezdi P, Zwart HHJ. The effect of allopurinol on the degree of early myocardial ischaemia. Am Heart J 1980;99:614-24.

${ }^{10}$ Taylor MD, Palmer GC, Callahan AS. Protective action of methyl prednisolone, allopurinol and indomethacin against stroke induced damage to adenylate cyclase in gerbil cortex. Stroke 1984;15:329-35. 